\title{
Protective effect of emodin on intestinal epithelial tight junction barrier integrity in rats with sepsis induced by cecal ligation and puncture
}

\author{
YANJUN LI $^{1 *}$, RUIMIN GUO ${ }^{*}$, MENGYING ZHANG $^{1}$, \\ PENG CHEN ${ }^{1}$, JINGXIN LI ${ }^{1}$ and YANNI SUN ${ }^{1,2}$ \\ ${ }^{1}$ Department of Emergency, Putuo Hospital, Shanghai University of Traditional Chinese Medicine; \\ ${ }^{2}$ Department of Emergency, Shanghai Putuo Central School of Clinical Medicine, \\ Anhui Medical University, Shanghai 200062, P.R. China \\ Received June 4, 2019; Accepted January 17, 2020
}

DOI: $10.3892 /$ etm.2020.8625

\begin{abstract}
The present study investigated the protective effects of emodin on intestinal epithelial tight junction (TJ) barrier integrity in cecal ligation and puncture (CLP)-induced septic rats and its possible mechanisms of action. Healthy male Sprague-Dawley rats were randomly divided into three groups ( $\mathrm{n}=20$ per group): Sham group, CLP group and CLP + emodin group. Animals were sacrificed at 12 and $24 \mathrm{~h}$ after the model was established. Abdominal aortic blood and specimens of the ileum were harvested for analysis. The histopathological changes in intestinal mucosa and the ultrastructures of intestinal epithelial cells were investigated using light microscopy and transmission electron microscopy. The integrity of the intestinal barrier was assessed by examining plasma diamine oxidase (DAO) levels and the ratio of urine lactulose to mannitol (L/M). The levels of the intestinal TJ proteins claudin-3, zonula occludens (ZO)-1 and occludin were detected using immunohistochemistry, western blotting and reverse transcription-quantitative PCR. The results showed that the pathological damage to intestinal mucosa and the intestinal tissue injury score in the CLP + emodin group were significantly reduced compared to those of the CLP group, and the differences were more obvious at $24 \mathrm{~h}$ compared with $12 \mathrm{~h}$. DAO activity and the L/M ratio in the emodin pre-treatment group decreased significantly at $24 \mathrm{~h}$ compared with the CLP groups. The protein and mRNA levels of the TJ proteins claudin-3, ZO-1 and occludin in the emodin
\end{abstract}

Correspondence to: Dr Yanni Sun, Department of Emergency, Putuo Hospital, Shanghai University of Traditional Chinese Medicine, 164 Lanxi Road, Putuo, Shanghai 200062, P.R. China E-mail: sunstone1974@163.com

*Contributed equally

Key words: cecal ligation and puncture, emodin, tight junction, claudin-3, zonula occludens-1, occludin pre-treatment groups at 12 and $24 \mathrm{~h}$ were increased, while occludin mRNA level was found to be decreased compared with the CLP groups. The present study suggested that emodin may significantly reduce the damage to the intestinal epithelial barrier in sepsis, inhibit intestinal barrier permeability and protect intestinal barrier integrity. Emodin may protect intestinal barrier integrity by elevating expression levels of the TJ proteins claudin-3, ZO-1 and occludin in CLP rats.

\section{Introduction}

Sepsis is a systemic inflammatory response that is caused by an overactive host response to infection (1). Although a series of advances have been made in medical therapy for sepsis, the mortality rate remains high (2) and is a challenge that has to be regularly overcome in the clinic (3). Sepsis causes excessive tissue damage and death in approximately 30-50\% of patients (4), and sepsis/septic shock are often accompanied by intestinal barrier dysfunction (5). Previous studies have revealed that intestinal barrier dysfunction plays an important role in the development of multiple organ dysfunction syndrome in sepsis $(6,7)$. Under normal physiological conditions, the intestinal epithelial barrier can effectively prevent viral microorganisms and endotoxins from transferring from the intestinal lumen to the blood (8). When sepsis occurs, an excessive inflammatory response contributes to intestinal epithelial cell apoptosis (9). Tight junctions (TJs) between epithelial cells are disrupted and paracellular gaps are widened (10), leading to impaired intestinal mucosal integrity. Furthermore, damage to the intestinal mucosa exacerbates bacterial translocation, leading to virulence factors that further activate the host immune inflammatory defence mechanisms, eventually resulting in multiple organ failure and life-threatening clinical symptoms (11). Destruction of the intestinal barrier integrity may be an important pathway which leads to the multiple organ dysfunction caused by sepsis (12). Protecting against intestinal epithelial barrier damage and maintaining intestinal epithelial barrier integrity reduces intestinal permeability and bacterial translocation. This protection is essential to improving the prognosis and survival of patients with sepsis (13). 
Rhubarb is used as a traditional Chinese medicine to treat intestinal diseases, and has been demonstrated to relieve intestinal damage in septic rats (14). Currently, a number of active ingredients have been extracted from rhubarb, including emodin, aloe-emodin, chrysophanol and physcion (15). It is not clear which of these components provides a protective effect on the intestinal mucosa in sepsis. Among them, emodin is the main effective component of rhubarb and is an anthraquinone derivative extracted from the rhizome of rhubarb (16). Emodin has numerous biological effects, such as antimicrobial, anti-inflammatory, anti-viral, antibacterial, anti-tumour and anti-fibrotic activities, as well as being able to affect diastolic blood pressure (17). Ning et al (18) showed that emodin can alleviate intestinal mucosal damage in experimental severe acute pancreatitis by inhibiting inflammatory cytokines, including interleukin (IL)-1 $\beta$ and IL-18. Wang et al (11), found that rhubarb monomers could promote the expression of the TJ proteins claudin-5, zonula occludens ( $\mathrm{ZO})-1$ and occludin and that this protected the intestinal mucosal barrier of rats with sepsis induced by cecal ligation and puncture (CLP). However, there is still no evidence showing the mechanism of action by which emodin protects the intestinal epithelial barrier integrity during sepsis. Since the integrity of the intestinal epithelial barrier is predominantly maintained by intestinal epithelial TJs, the mechanism by which the intestinal epithelial barrier can be improved has been a focus of research (19). Various TJ proteins are expressed differently in intestinal tissue. The mRNA expression levels of claudin-3 are higher in the duodenum than the distal colon, while claudin-5 shows the reverse relationship $(20,21)$. In the present study, it was hypothesized that emodin provides a protective function on intestinal epithelial TJ barrier integrity in CLP-induced septic rats. A possible mechanism was related to the expression of the TJ proteins claudin-3, ZO-1 and occludin.

\section{Materials and methods}

Ethics approval. The present study was approved by the Ethics Committee of Putuo Hospital affiliated with Shanghai University of Traditional Chinese Medicine. Specific pathogen-free male Sprague-Dawley rats $(n=60)$ weighing $280 \pm 20 \mathrm{~g}$ and aged $8-10$ weeks were obtained from the Shanghai Slack Laboratory Animal Co., Ltd. All rats were acclimatized in housing conditions at $24 \pm 2^{\circ} \mathrm{C}, 50 \%$ humidity and $12 \mathrm{~h}$ light-dark cycles. Rats were fed with conventional laboratory feed and water ad libitum. All animal experiments were in accordance with the Experimental Animal Centre Committee of Putuo Hospital (certification no. SYNK 2018-0032).

Animals and experimental design. Following the random number table method, rats were divided into the sham group, CLP group and CLP + emodin group, with 20 rats in each group. The CLP + emodin group was intragastrically administered with $35 \mathrm{mg} \mathrm{kg}^{-1}$ emodin [high-performance liquid chromatography (HPLC) $\geq 98 \%$; Shanghai Gefan Biotechnology Co., Ltd.] in a $0.5 \%$ sodium carboxy-methylcellulose suspension (prepared to contain emodin at $5 \mathrm{~g} \mathrm{~L}-1$ ) once daily at 09:00 a.m., for 5 days (22). The sham and CLP groups were intragastrically administered an equal amount of $0.5 \%$ sodium carboxy-methylcellulose solution. Following $2 \mathrm{~h}$ after the last gavage, the sepsis model was induced in the CLP and CLP + emodin groups. The sham group was treated as previously described (23), briefly: The rats were anaesthetized, and a $1 \mathrm{~cm}$ incision was made along the midline of the abdomen. The caecum was removed, and the caecum was returned to the abdominal cavity 2 min later. The CLP groups were treated as previously described (24), briefly: The rats were fasted for $12 \mathrm{~h}$ before surgery. The rats were then fixed on a console and were anaesthetized by intraperitoneal injection of $2.5 \%$ sodium pentobarbital $\left(30 \mathrm{mg} \mathrm{kg}^{-1}\right)$. An incision of $\sim 1 \mathrm{~cm}$ was cut along the midline of the abdomen, the mesentery and caecum were separated, and the caecal root was ligated using a 4/0 silk thread. A $1 \mathrm{~cm}$ syringe needle was used to puncture the cecum at the ligation site, a small amount of faeces was squeezed out, and the peritoneum and skin were stitched. Finally, 10 rats from each group were sacrificed at $12 \mathrm{~h}$ and $24 \mathrm{~h}$ after model establishment. Prior to sacrifice, all rats were anesthetized using an intraperitoneal injection of $2.5 \%$ sodium pentobarbital $\left(50 \mathrm{mg} \mathrm{kg}^{-1}\right)$. Rats were sacrificed by cervical dislocation immediately following blood collection. The blood was collected from the abdominal aorta, and the ileum tissues were separated from the ileocecal area.

Haematoxylin and eosin $(H \& E)$ staining for histopathology. The ileum tissues were fixed with $10 \%$ formalin at room temperature for 7 days. The samples were dehydrated, embedded in paraffin, sliced into $4-\mu \mathrm{m}$ sections and stained with H\&E. Samples were stained with hematoxylin for $10 \mathrm{~min}$ and stained with eosin for $2 \mathrm{~min}$ at room temperature. Subsequently, the sections were examined under a light microscope at magnification, $x 400$. The images were observed by two pathologists who were blinded to the experimental conditions of the present study. Intestinal mucosal injuries were assessed using Chiu's score grading (25).

Transmission electron microscopy. A small segment of the ileum was spread on filter paper to make a specimen of $1.5 \times 1.5 \mathrm{~cm}$ and fixed with $2.5 \%$ glutaraldehyde at $4^{\circ} \mathrm{C}$ for 7 days. Then, in conjunction with the Electron Microscopy Testing Centre of Shanghai University of Traditional Chinese Medicine, the ileum specimens were adjusted to $\sim 1 \mathrm{~mm}^{3}$, incubated with $1 \%$ osmium acid at room temperature for $2 \mathrm{~h}$, and finally dehydrated with ethanol and acetone. The fixed tissue was embedded in epoxy resin and cut into 50-nm ultrathin sections. The sections were then stained with uranyl acetate at room temperature for $2 \mathrm{~h}$. The tissue sections were observed under a transmission electron microscope and images were captured at magnification, $x 8,200$ to determine whether the microvilli arrangement on the surface of intestinal mucosal epithelial cells and the ultrastructures of organelles such as epithelial space, TJs and mitochondria in epithelial cells were normal.

Detection of diamine oxidase (DAO) activity in intestinal tissue. Blood taken from the abdominal aorta of each rat was centrifuged at $1,006.2 \mathrm{x} \mathrm{g}$ at $4^{\circ} \mathrm{C}$ for $15 \mathrm{~min}$. The supernatant was collected and stored at $-80^{\circ} \mathrm{C}$ until examination. The activity of DAO was determined using a DAO kit (Nanjing Jiancheng Bioengineering Institute). The procedure followed 
the manufacturer's protocol. Optical density was measured using a microplate reader at $340 \mathrm{~nm}$ within $20 \mathrm{sec}$. A standard curve was used to calculate the activity of DAO and to statistically analyse the data.

Detection of the urine lactulose/mannitol (L/M) ratio in intestinal tissue. The rats were given a mannitol (HPLC $\geq 98 \%$; Shanghai Gefan Biotechnology Co., Ltd.) and lactulose (HPLC $\geq 98 \%$; Shanghai Gefan Biotechnology Co., Ltd.) suspension [50 mg mannitol and $100 \mathrm{mg}$ lactulose dissolved in $2 \mathrm{ml}$ double distilled (dd) $\mathrm{H}_{2} \mathrm{O}$ ], administered intragastrically $2 \mathrm{ml} / \mathrm{rat}, 2 \mathrm{~h}$ after establishing the sepsis model. Urine was collected within $6 \mathrm{~h}$ and was centrifuged at 8,049.6 $\mathrm{x} \mathrm{g}$ at $4^{\circ} \mathrm{C}$ for $4 \mathrm{~min}$. The supernatant was stored at $-80^{\circ} \mathrm{C}$ and analyzed by mass spectrometry in collaboration with the Shanghai University of Traditional Chinese Medicine Science and Technology Innovation Centre. Standards were prepared by diluting mannitol, lactulose and deuterated mannitol with $\mathrm{ddH}_{2} \mathrm{O}$ to varying concentrations. The samples were diluted 10 times with $\mathrm{ddH}^{2} \mathrm{O}$; then, $50 \mu \mathrm{l}$ of sample was added to $450 \mu \mathrm{l}$ of acetonitrile (containing $1 \mu \mathrm{g} \mathrm{ml}^{-1}$ internal standard), vortexed at room temperature for $1 \mathrm{~min}$, and centrifuged at $14,310.4 \mathrm{x} \mathrm{g}$ at $4^{\circ} \mathrm{C}$ for $5 \mathrm{~min}$. A total of $10 \mu \mathrm{l}$ of the supernatant was sampled. The instrument parameters were set, including the mass spectrometry conditions. Electrospray ion source nozzle position was 3:7, atomizing gas flow rate was $101 \mathrm{~min}^{-1}$, gas curtain gas flow rate was $121 \mathrm{~min}^{-1}$, collision gas flow rate was $121 \mathrm{~min}^{-1}$, ion source voltage was $-4500 \mathrm{~V}$, ion source temperature was $300^{\circ} \mathrm{C}$, and ion selective channel $\mathrm{m} / \mathrm{z}$ (mass to charge ratio) were as follows: Lactulose, 341.1/160.7 (collision energy, -13; declustering potential, -74); mannitol, 181.1/88.9 (collision energy, -21; declustering potential, -70); and deuterated mannitol, 183.0/88.9 (collision energy, -21; declustering potential, -67). Isocratic elution at a flow rate of $0.3 \mathrm{ml} \mathrm{min} \mathrm{m}^{-1}$ and a volume of $10 \mu \mathrm{l}$ per sample were used. The chromatogram was recorded and the sample content was calculated from the standard curve. Finally, the urine L/M ratio and statistics were calculated.

Immunohistochemistry. Ileum tissue was fixed in $10 \%$ formalin at room temperature for 7 days. The tissue pieces were cut and embedded in paraffin. Sections were cut to a thickness into $5-\mu \mathrm{m}$ and placed on slides. The samples were dehydrated by gradient alcohol. The alcohol concentration was 100, 95, 90, $80,70,50$, and $30 \%$, and dehydration was performed for $\sim 35$ min at room temperature. A $1 \%$ sodium citrate antigen repair solution was used to soak the slides in a $100^{\circ} \mathrm{C}$ water bath for $30 \mathrm{~min}$. When cooled to room temperature, the samples were incubated with $3 \%$ hydrogen peroxide for $10 \mathrm{~min}$ and rinsed with 1x PBS (3x5 min) before incubation with 5\% BSA at $37^{\circ} \mathrm{C}$ for $10 \mathrm{~min}$. Rabbit antibodies against ZO-1 (1:100; cat. no. 40-2200; Invitrogen; Thermo Fisher Scientific, Inc.), occludin (1:80; cat. no. ab216327; Abcam) and claudin-3 (1:80; cat. no. ab15102; Abcam) )were added and incubated at $4{ }^{\circ} \mathrm{C}$ overnight. The slides were washed with 1x PBS (3x5 min), and horseradish peroxidase conjugated anti-rabbit immunoglobulin G secondary antibody (cat. no. SA1022; Boster Biological Technology) was added and incubated at $37^{\circ} \mathrm{C}$ for $30 \mathrm{~min}$. The slides were washed with $1 \mathrm{X}$ PBS $(3 \times 5 \mathrm{~min})$, StreptAvidin-Biotin Complex was added and incubated at $37^{\circ} \mathrm{C}$ for $30 \mathrm{~min}$. The slides were washed with $1 \mathrm{X}$ PBS ( $3 \times 5 \mathrm{~min})$, and 3,3'-diaminobenzidine dye was added at room temperature for 1-3 sec under a fluorescent microscope. The slides were washed with $1 \mathrm{X}$ PBS ( $3 \times 5 \mathrm{~min})$ and counterstained with haematoxylin at room temperature for $\sim 15 \mathrm{sec}$. Then, the slides were dehydrated with gradient alcohol and the alcohol concentration was 30, 50, 70, 80, 90, 95 and $100 \%$, and dehydration was performed for $20 \mathrm{~min}$ at room temperature. Xylene was subsequently added to the slides at room temperature for $\sim 10$ min, glass coverslips were added and images were captured with a fluorescent microscope (magnification, x200).

Western blot analysis. Ileum tissues were removed from $-80^{\circ} \mathrm{C}$ storage and cut into 50-mg pieces. A total of $1 \mathrm{ml}$ protein lysis buffer (1:1) composed of RIPA buffer (cat. no. P0013C; Beyotime Institute of Biotechnology) and EDTA-free protease inhibitor cocktail (cat. no. 04693132001; Roche; Merck KGaA) was added to extract the proteins using a tissue homogenizer. The supernatant was then collected by centrifugation at $8,049.6 \mathrm{x} \mathrm{g}$ at $4^{\circ} \mathrm{C}$ for $5 \mathrm{~min}$. Total protein was determined using a bicinchoninic acid protein quantitation kit (cat. no. P0010S; Beyotime Institute of Biotechnology). Samples were mixed with 1X SDS-PAGE protein loading buffer (cat. no. P0015; Beyotime Institute of Biotechnology) and denatured by heating at $100^{\circ} \mathrm{C}$ for $15 \mathrm{~min}$. Next, the samples $(60 \mu \mathrm{g}$ protein) were separated on SDS-PAGE with $10 \%$ gel (cat. no. P0012A; Beyotime Biotechnology) until the bromophenol blue had migrated out of the end of the gel. Proteins were then transferred on to a PVDF membrane (EMD Millipore) and blocked in 5\% milk at room temperature for $2 \mathrm{~h}$. The membranes were incubated overnight with rabbit antibodies against GAPDH $(0.925 \mathrm{mg} / \mathrm{ml} ; 1: 3,000$ dilution; cat. no. ab181602; Abcam), ZO-1 (0.25 mg/ml; 1:1,000 dilution; cat. no. 40-2200; Invitrogen; Thermo Fisher Scientific, Inc.), occludin (0.604 mg/ml, 1:500 dilution; cat. no. ab216327; Abcam), and claudin-3 (500 $\mu 1,1: 1,000$ dilution; cat. no. ab15102; Abcam). The membranes were washed with $1 \mathrm{X}$ TBS-Tween-20 (TBS-T; 0.1\% Tween-20; 3x10 min) and placed in goat anti-rabbit secondary antibody (1:2,000 dilution, cat. no. 111-005-003; Jackson ImmunoResearch Laboratories, Inc.) at room temperature for $2 \mathrm{~h}$. The membranes were washed with 1X TBST (3x10 min) and developed using the Clarity Western ECL substrate (cat. no. 170-5060; Bio-Rad Laboratories, Inc.). Finally, the density of the bands was quantified using ImageJ software (version 1.51; National Institutes of Health). Data were normalized against a housekeeping gene GAPDH and the expression levels of the other groups were presented as the relative fold difference compared to the sham group (26).

Reverse transcription-quantitative PCR (RT-qPCR). The ileum tissues were removed from $-80^{\circ} \mathrm{C}$ storage and cut into 50 -mg pieces. Total RNA was extracted with TRIzol ${ }^{\circledR}$ reagent (cat. no. 9109; Takara Biotechnology Co., Ltd.) following the manufacturer's instructions. The concentration of the RNA samples was determined using spectrophotometric optical density measurement at $260 \mathrm{~nm}$. A total of $1 \mu \mathrm{g}$ of total RNA from each sample was reverse transcribed into cDNA using a PrimeScript RT Reagent kit (cat. no. RR047A; Takara Bio, Inc.). The following heat cycle was used for RT: $42^{\circ} \mathrm{C}$ for $2 \mathrm{~min}$, $37^{\circ} \mathrm{C}$ for $15 \mathrm{~min}$ at and $85^{\circ} \mathrm{C}$ for $5 \mathrm{sec}$. PCR was performed 
Table I. Primers used in the present study.

Primer sequence

Gene [NCBI reference]

Forward

Reverse

ZO-1 [NM_001106266]

occludin [NM_031329]

claudin-3 [NM_031700]

$\beta$-actin [NM_031144]

\author{
5'-CAGGCCATTACGAGCCTCTC-3' \\ 5'-CCAATGGCCTACTCCTCCAA-3' \\ 5'-ATTCATCGGCAGCAGCATC-3' \\ 5'-TGTCACCAACTGGGACGATA-3'
}

5'-AGGCTGTGGCTTGGTAGCTG-3'
5'-CATCCACGGACAAGGTCAGA-3'
5'-CCAGCAGCGAGTCGTACATC-3'
5'-GGGGTGTTGAAGGTCTCAAA-3'

ZO, zonula occludens.

using a PCR machine (Applied Biosystems; Thermo Fisher Scientific, Inc.). Each reaction volume consisted of $20 \mu \mathrm{l}$ and contained $5 \mu \mathrm{l}$ of sample cDNA, $10.4 \mu \mathrm{l}$ TB Green Premix Ex Taq PCR master mix (cat. no. RR420A; Takara Bio, Inc.),

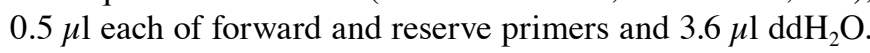
$\beta$-actin was used as a reference gene. The primer sequences of the target genes are shown in Table I.

The PCR conditions were $1 \mathrm{~min}$ at $95^{\circ} \mathrm{C}$, followed by 40 cycles of $5 \mathrm{sec}$ at $95^{\circ} \mathrm{C}$ and $30 \mathrm{sec}$ at $60^{\circ} \mathrm{C}$. The last stage was followed by an incubation of $15 \mathrm{sec}$ at $95^{\circ} \mathrm{C}, 1 \mathrm{~min}$ at $60^{\circ} \mathrm{C}$ and $15 \mathrm{sec}$ at $95^{\circ} \mathrm{C}$ to establish a PCR melting curve. Relative quantification of PCR products was normalized against the sham group to calculate the relative expression in the CLP group and CLP + emodin group. Relative expression levels of ZO-1, occludin, and claudin-3 mRNA levels were analyzed using the 2- ${ }^{\Delta \Delta \mathrm{Cq}}$ method (27) with QuantStudio_Flex Real-Time PCR System software (version 1.3; Thermo Fisher Scientific, Inc.).

Statistical analysis. All statistical analyses were performed using SPSS software (version 24.0; IBM Corp.), and the measurement data are expressed as the mean \pm SD. All data were tested for homogeneity of variances. One-way ANOVAs were used for comparisons between groups. Fisher's least significant difference test was used for multiple comparisons between groups. The pathological scores were compared using Kruskal-Wallis test with post-hoc Nemenyi tests for multiple comparisons. $\mathrm{P}<0.05$ was considered to indicate a statistically significant difference.

\section{Results}

Emodin alleviates the pathological changes of intestinal epithelial TJ barrier injury in septic rats

Emodin relieves the pathological damage to the intestinal epithelial barrier in septic rats. As observed by light microscopy, the sham group (Fig. 1A) had intact intestinal mucosa, and the intestinal villi were neatly arranged. After CLP, the intestinal mucosa of the CLP group (Fig. 1B) presented different degrees of congestion and oedema, inflammatory cell infiltration, irregular arrangement of intestinal villi and an enlarged villus gap. The intestinal mucosa apical epithelial space was further enlarged, the top epithelium of the villus was detached, and the intestinal lamina propria was disrupted. Compared with the damage in the CLP group, the emodin pre-treatment group (Fig. 1C) showed reduced intestinal mucosal damage. Using the Chiu scoring system to semi-quantitatively analyse the intestinal mucosal morphology of each group, it was found that the CLP group had a significantly higher score than that of the sham group, and the damage at $24 \mathrm{~h}$ in the model group was the highest $(\mathrm{P}<0.01 ; 12$ and $24 \mathrm{~h}$, respectively; Fig. 1D). After pre-treatment with emodin, the CLP + emodin group had a significantly lower score compared with the CLP model group $(\mathrm{P}<0.01 ; 12$ and $24 \mathrm{~h}$, respectively). These data indicated that emodin may alleviate the pathological damage to intestinal mucosa in rats with sepsis caused by CLP.

Emodin improves the pathological changes of $\mathrm{TJ}$ barrier ultrastructures in septic rats. Electron microscopy showed that in the sham operation group (Fig. 2A), the intestinal epithelial cells were closely arranged, the microvilli on the surface of epithelial cells were neatly arranged; the TJ stents and desmosomes were clear and intact, and the paracellular spaces were narrow. However, in the CLP group (Fig. 2B), the microvilli on the surface of intestinal epithelial cells were unevenly arranged, the lengths were irregular and there were different degrees of shedding. In addition, the gaps between epithelial cells were widened, and the boundaries of the TJ scaffolds and desmosomes unclear or had disappeared. After pre-treatment with emodin (Fig. 2C), the TJ gaps were significantly reduced, the epithelial cells were tightly connected and microvilli injury appeared to be alleviated. These results indicated that emodin alleviated the sepsis-induced pathological changes in the ultrastructures of the intestinal TJ barrier.

Emodin maintains the integrity of the intestinal epithelial barrier in sepsis by reducing serum DAO levels. The plasma DAO results (Fig. 3A) indicated that compared with the corresponding sham groups, the DAO levels in the CLP groups increased significantly $(\mathrm{P}<0.05 ; 12$ and $24 \mathrm{~h}$, respectively) and peaked at $24 \mathrm{~h}$. After emodin intervention, the activity of DAO was significantly lower in the CLP + emodin treatment group when compared with that of the corresponding CLP groups at 12 and $24 \mathrm{~h}(\mathrm{P}<0.05 ; 12$ and $24 \mathrm{~h}$, respectively). The above data indicated that emodin could inhibit the increase in DAO activity in the serum of rats with CLP-induced sepsis and had a protective effect in intestinal mucosal injury.

Emodin maintains the integrity of the intestinal epithelial barrier in sepsis by reducing the L/M ratio in urine. The results of high-performance liquid chromatography-based 


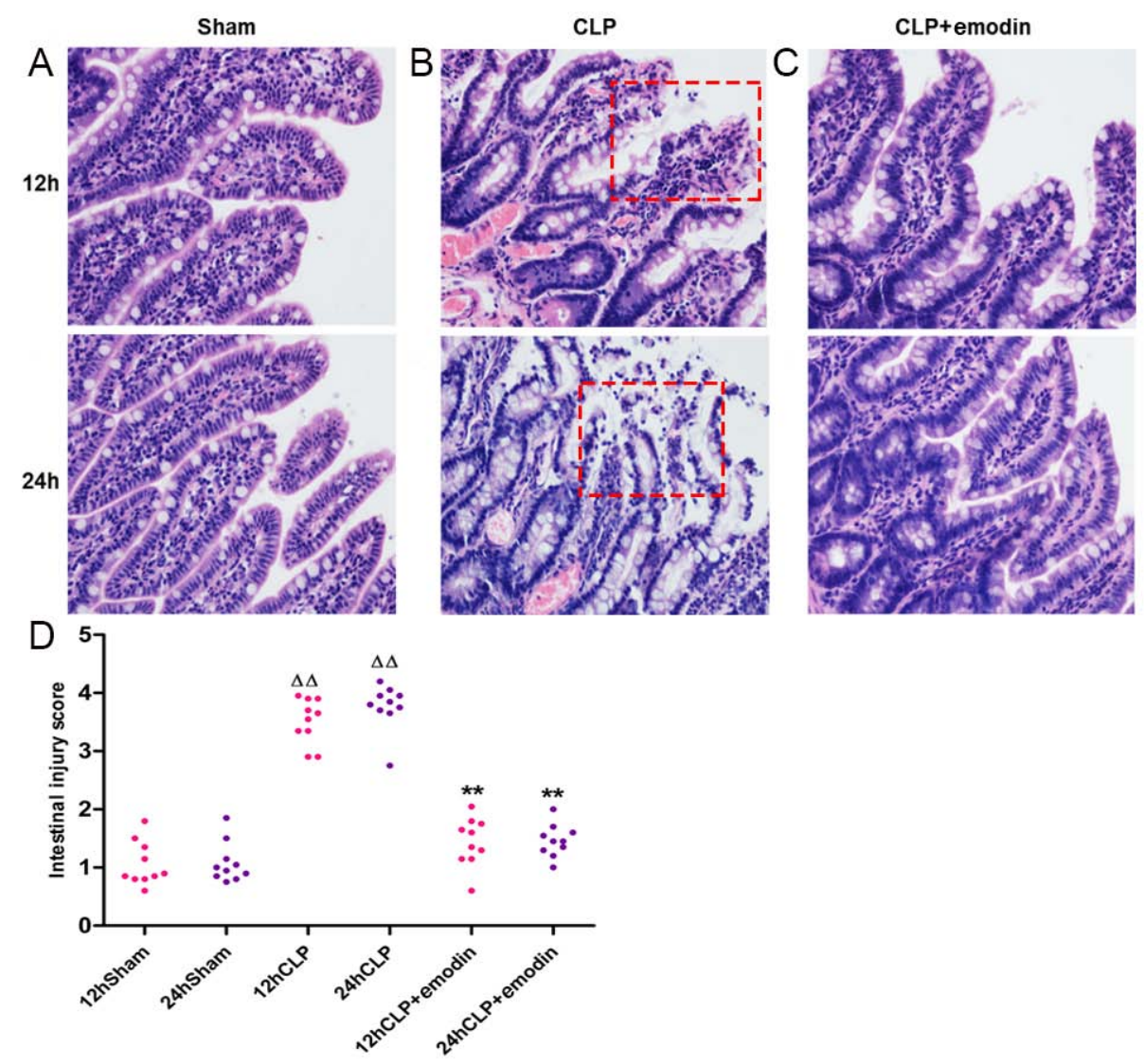

Figure 1. Histological assessment of the effects of emodin on intestinal tissue of CLP-induced septic rats. Representative images of hematoxylin and eosin-stained intestinal sections at 12 and $24 \mathrm{~h}$ after CLP operation for (A) the sham group, (B) the CLP group and (C) the CLP + emodin group. (D) Intestinal tissue Chiu's scores are shown. The intestinal mucosa of the CLP group (red dotted box) had severe congestion, oedema, inflammatory cell infiltration and widened intestinal villus. Further enlargement or shedding of intestinal epithelial space were evident along with a high Chiu's score compared with those of the sham group. Emodin had a therapeutic effect in the CLP + emodin group. The data are presented as the mean \pm standard deviation. ${ }^{* *} \mathrm{P}<0.01 \mathrm{vs}$. the corresponding CLP group; ${ }^{\Delta} \mathrm{P}<0.01$ vs. the corresponding sham group. Magnification, $\mathrm{x} 400$. CLP, cecal ligation and puncture.

mass spectrometry for the determination of mannitol and lactulose in urine (Fig. 3B) showed that the urine L/M ratio in the sham group did not change significantly with time. In the CLP group, the L/M ratio was significantly increased compared with that of the sham group ( $\mathrm{P}<0.01 ; 12$ and $24 \mathrm{~h}$, respectively). Compared with the L/M ratio in the CLP group, the L/M ratio in the CLP + emodin group was significantly reduced at $24 \mathrm{~h}$ $(\mathrm{P}<0.05 ; 24 \mathrm{~h})$. These data suggested that emodin could inhibit the increase in intestinal epithelial barrier permeability in septic rats and help to maintain the integrity of the epithelial barrier.

Emodin promotes the expression of the TJ protein claudin-3 in the intestinal epithelium of CLP-induced septic rats. As shown in Fig. 4, immunohistochemical microscopy images showed the expression of claudin-3 in the ileums of septic rats (Fig. 4A). In the sham group, claudin-3 expression (brown) was uniformly distributed in the intestinal epithelial cells at the apex of each connection and it was tightly packed with smooth edges. Claudin-3 was unevenly distributed, with rough edges and faded staining in the CLP group. However, in the CLP + emodin group, claudin-3 staining was darker and continuously distributed. Western blotting (Fig. 4D) and RT-qPCR analysis (Fig. 4E) showed that compared with the expression levels in the sham group, the expression of claudin-3 was decreased in the CLP group, and there was a significant difference at $24 \mathrm{~h}(\mathrm{P}<0.01$ in the western blotting and RT-qPCR, respectively). With the emodin intervention, claudin-3 protein levels were significantly increased in the emodin pre-treatment groups at 12 and $24 \mathrm{~h}$ compared to the CLP treatment alone $(\mathrm{P}<0.01$ and $\mathrm{P}<0.05 ; 12$ and $24 \mathrm{~h}$, respectively). The trend in claudin-3 mRNA levels was consistent with that of the protein levels $(\mathrm{P}<0.05$ and $\mathrm{P}<0.01 ; 12$ and $24 \mathrm{~h}$, respectively). These data indicated that emodin could inhibit the increase in intestinal barrier permeability in sepsis by promoting the expression of claudin-3 protein and mRNA.

Emodin promotes the expression of the TJ protein $\mathrm{ZO}-1$ in the intestinal epithelium of CLP-induced septic rats. As depicted in Fig. 4, immunohistochemical microscopy images showed ZO-1 expression in the ileums of septic rats (Fig. 4B). In the sham group, ZO-1 expression (brown) was continuously and evenly distributed as well as being tightly packed, with smooth edges and a strong positive expression. ZO-1 was unevenly distributed, with rough edges and faded staining in the CLP group. However, in the CLP + emodin group, ZO-1 staining was darker and presented a continuous distribution. Western blotting (Fig. 4D) and RT-qPCR analysis (Fig. 4F) showed that ZO-1 protein expression at $12 \mathrm{~h}(\mathrm{P}<0.05)$ and mRNA levels at $24 \mathrm{~h}(\mathrm{P}<0.01)$ were significantly decreased in the CLP group 

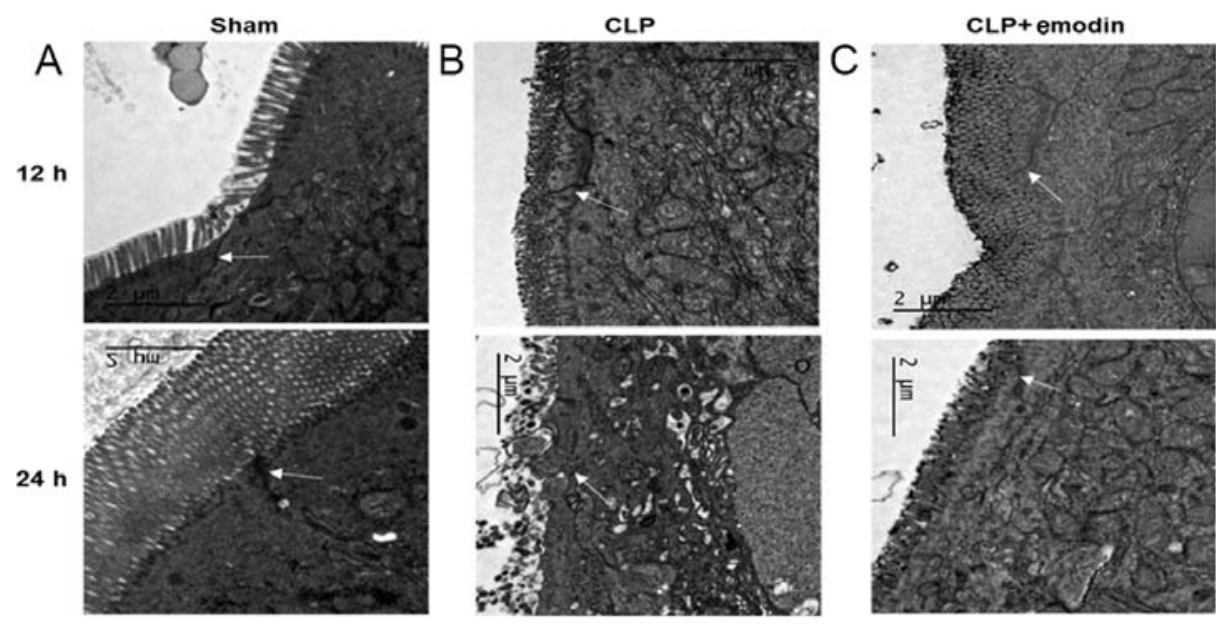

Figure 2. Transmission electron microscopy images of tight junction structural morphology in ileal mucosa. (A) Sham control group; (B) CLP group; (C) CLP + emodin group. White arrows indicate tight junctions. Magnification, x8,200. CLP, cecal ligation and puncture.

A

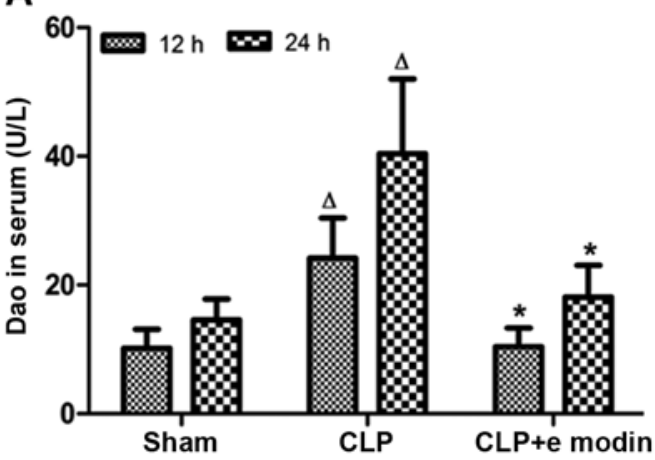

B

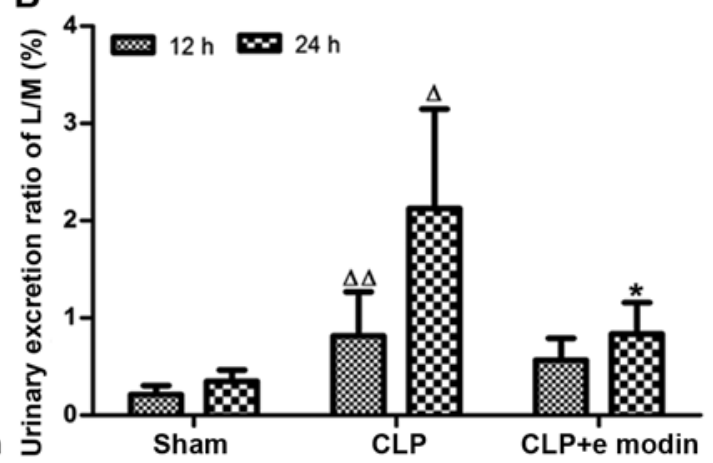

Figure 3. Effects of emodin on intestinal barrier permeability in CLP-induced sepsis rats. (A) Effect of emodin on serum DAO levels in CLP-induced septic rats. DAO levels were analyzed using enzymatic spectrophotometry. (B) Effect of emodin on the L/M ratio in the urine of CLP-induced septic rats. Urine $\mathrm{L} / \mathrm{M}$ ratios were analyzed using high-performance liquid chromatography-based mass spectrometry. Data are expressed as the mean $\pm \mathrm{SD}$. ${ }^{*} \mathrm{P}<0.05 \mathrm{vs}$. the corresponding CLP group; ${ }^{\Delta \mathrm{P}}<0.05$ and ${ }^{\Delta}{ }^{\mathrm{P}}<0.01$ vs. the corresponding sham group. CLP, cecal ligation and puncture; DAO, diamine oxidase; $\mathrm{L} / \mathrm{M}$, lactulose to mannitol.

compared with the sham group. After emodin intervention, the protein expression levels of ZO-1 were augmented in the emodin pre-treatment groups at 12 and $24 \mathrm{~h}$ compared with the CLP treatment alone group, and there was a statistically significant difference $(\mathrm{P}<0.01 ; 12$ and $24 \mathrm{~h}$, respectively). The mRNA levels of ZO-1 were consistent with that of the protein expression $(\mathrm{P}<0.05$ and $\mathrm{P}<0.01 ; 12$ and $24 \mathrm{~h}$, respectively). These results indicated that emodin may inhibit the increase in intestinal barrier permeability in sepsis by promoting the expression of ZO-1.

Emodin partially promotes expression of the intestinal epithelial TJ protein occludin in CLP-induced septic rats. As depicted in Fig. 4, immunohistochemical microscopy images showed occludin expression in the ileums of septic rats (Fig. 4C). In the sham group, occludin expression (brown) was uniformly distributed in the intestinal epithelial cells at the apex of each connection and tightly packed within smooth edges. Occludin was unevenly distributed, with rough edges and darker colours in the CLP group. However, in the CLP + emodin group, occludin staining was darker and with a continuous distribution. Western blot assays showed that compared with the sham group, there was no obvious change in the expression levels in the CLP group (Fig. 4D). RT-qPCR analysis showed that compared with levels in the sham group, the levels of occludin mRNA were significantly raised in the CLP group at $12 \mathrm{~h}(\mathrm{P}<0.01$; Fig. 4G). After emodin pre-treatment, the protein levels of occludin were increased in the CLP + emodin group, and there was a significant difference at $12 \mathrm{~h}(\mathrm{P}<0.01)$. However, occludin mRNA was significantly decreased at $12 \mathrm{~h}$ and $24 \mathrm{~h}(\mathrm{P}<0.01)$. These data suggested that emodin can inhibit the increase in intestinal barrier permeability in sepsis by promoting the expression of occludin protein.

\section{Discussion}

The present study confirmed the protective effect of emodin on CLP-induced intestinal epithelial barrier injury in sepsis. It was found that emodin inhibited the increase in intestinal barrier permeability induced by CLP. Emodin may also maintain the integrity of the intestinal epithelial TJ barrier by promoting the expression of TJ proteins, including claudin-3, ZO-1 and occludin. CLP-induced sepsis is the most commonly used animal modelling method for sepsis (28). This model 
A
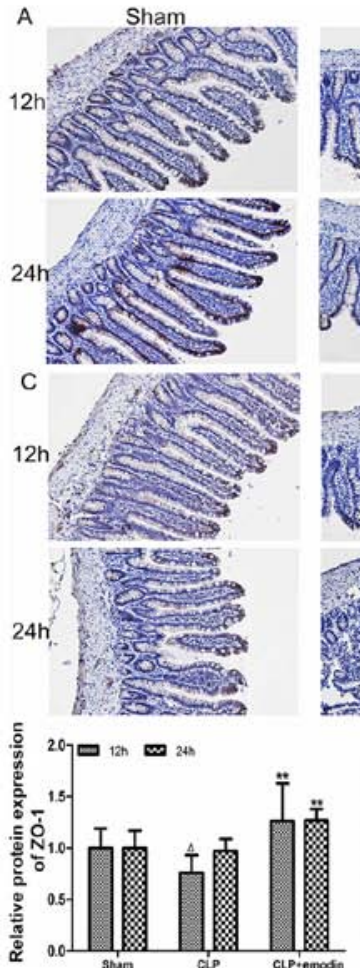

CLP
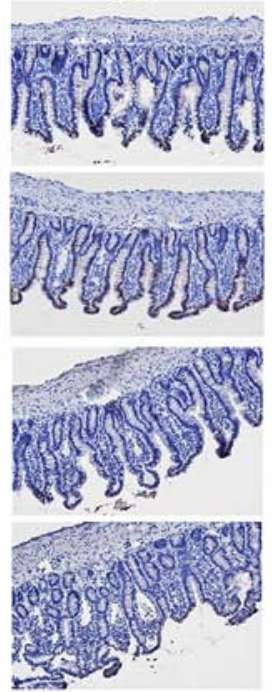
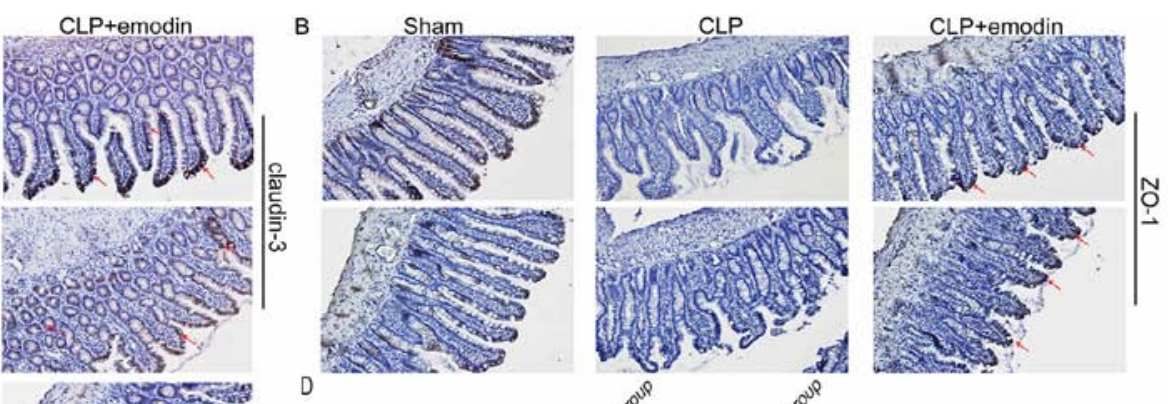
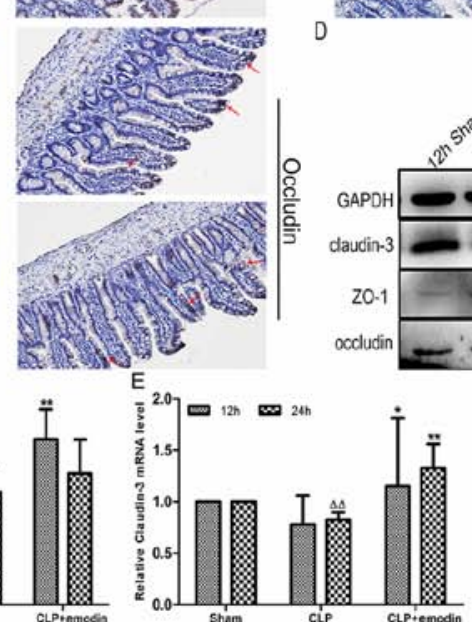
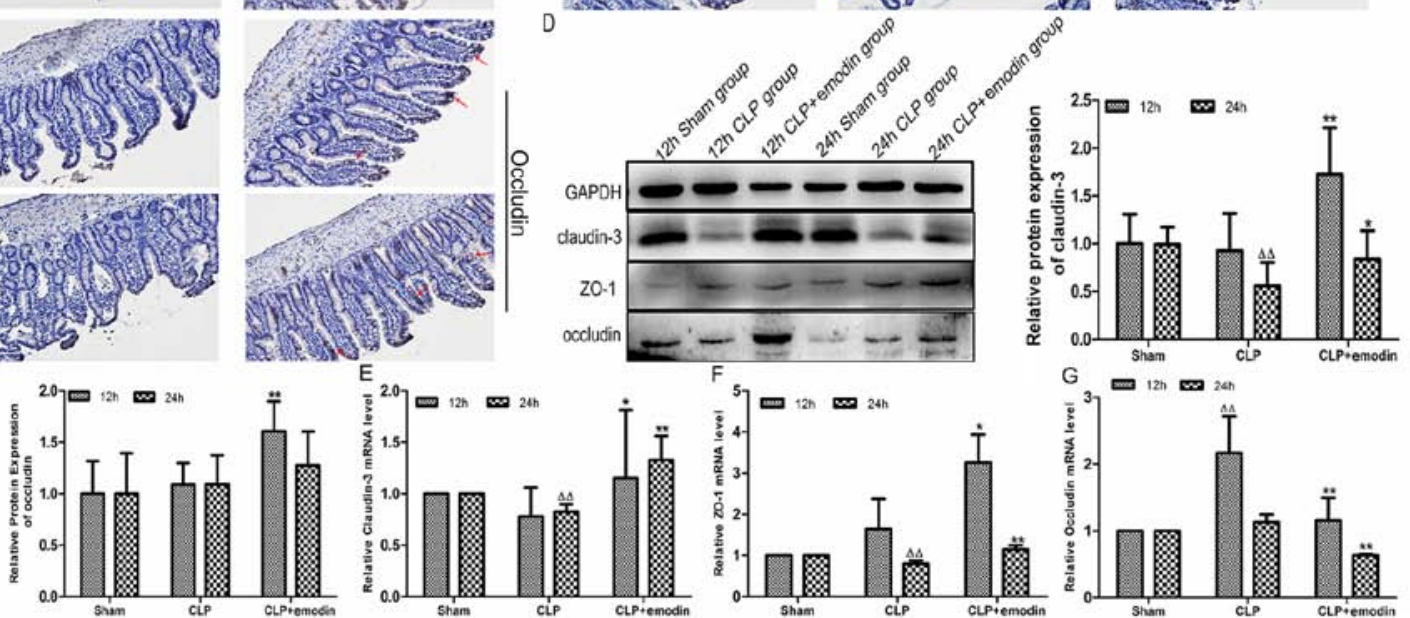

Figure 4. Regulation of intestinal tight junction proteins by emodin in CLP-induced septic rats. Immunohistochemical microscopy images showing the expression of (A) claudin-3, (B) ZO-1 and (C) occludin in the ileums of septic rats. Claudin-3, ZO-1 and occludin (brown) showed intense staining in the sham group ileum tissue nucleus. (blue); however, these proteins were less frequently detected in the CLP group. Moreover, expression was markedly increased in the CLP rats that were treated with emodin. (D) Western blot assays showing that the expression levels of claudin-3, ZO-1 and occludin. Reverse transcription-quantitative PCR analysis showed that the mRNA levels of (E) claudin-3, (F) ZO-1 and (G) occludin in the ileums of septic rats. Data are presented as the mean \pm SD. ${ }^{*} \mathrm{P}<0.05$ and ${ }^{* *} \mathrm{P}<0.01$ vs. the corresponding CLP group; ${ }^{\Delta} \mathrm{P}<0.05$ and ${ }^{\Delta \Delta} \mathrm{P}<0.01$ vs. the corresponding sham group. Red arrows indicate where the expression of tight junction protein is more apparent. Magnification, $\mathrm{x} 200$. CLP, cecal ligation and puncture; ZO-1, zonula occludens-1.

accurately represents the progression and properties of human sepsis. The model has a haemodynamic and metabolic phase similar to abdominal infections, accompanied by the presence of different stages of inflammation, as well as a prolonged and reduced cytokine release (11).

In the present study, CLP was used to construct a classical model of septic intestinal injury. The results showed that the intestinal mucosa appeared to have different degrees of pathological changes in the CLP group at 12 and $24 \mathrm{~h}$ following treatment, including intestinal epithelial inflammatory cell infiltration, intestinal villi that were not aligned and even the occurrence of shedding, all of which were consistent with previous reports $(29,30)$. However, it was found that the intestinal mucosal injury score of the $24 \mathrm{~h}$ CLP group was higher than that of the $12 \mathrm{~h}$ CLP group, and the pathological changes in the intestinal mucosa were more severe at $24 \mathrm{~h}$. The reason for this phenomenon may be related to the CLP modelling method. With the progression of CLP, the bacteria and toxins in the intestinal cavity invade distant organs and the circulatory system through the portal system, the intestinal lymphatic pathway, and the direct osmotic reabsorption pathway at $24 \mathrm{~h}(31,32)$. The liver and lungs receive venous blood and lymph, respectively, from the gastrointestinal tract. Inflammatory effectors are activated by intestinal bacteria and toxins, releasing cytokines and inflammatory mediators, which in turn further stimulates serious systemic inflammation and aggravates intestinal mucosal damage (14). DAO is an important indicator for measuring intestinal mucosal damage, and a reduction in DAO indicates that the integrity of the intestinal mucosa is destroyed (33). Lactulose is a disaccharide with a molecular weight of 342 , and mannitol is a monosaccharide with a molecular weight of 182 . Both of these sugars are not easily metabolised in the body. Generally, lactulose and mannitol are excreted in the urine and can be accurately quantified. The urine $\mathrm{L} / \mathrm{M}$ ratio is a classic method for detecting intestinal barrier function and intestinal barrier permeability (34). In the present study, DAO levels and the L/M ratio were examined to evaluate the integrity of the intestinal barrier in sepsis. The results reflected that DAO levels and the $\mathrm{L} / \mathrm{M}$ ratio were increased in the CLP group at 12 and $24 \mathrm{~h}$. The DAO levels in the $24 \mathrm{~h}$ CLP group were markedly higher than those at $12 \mathrm{~h}$. This trend was consistent with the extent of intestinal mucosal damage. These results also indirectly confirmed the hypothesis that intestinal mucosal damage is more severe at $24 \mathrm{~h}$ after CLP compared to at $12 \mathrm{~h}$.

Emodin has been shown to reduce septic jejunal damage by inhibiting apoptosis of intestinal epithelial cells through anti-inflammatory effects (35). In the present study, light microscopy and electron microscopy were used to observe the pathological changes in the intestinal mucosal epithelium. The results showed that emodin can significantly reduce the pathological damage to intestinal mucosa and reduce the Chiu's ileal score. Under light microscopy, the intestinal mucosal tissue showed oedema and was infiltrated with inflammatory cells. The structure of intestinal villi was also complete. Electron microscopy showed that the microvilli on the surface of the 
intestinal epithelium were neatly arranged, the TJ structures of the epithelial cells were intact, the gaps were narrowed and the junctions were tight. In addition, Chiu's intestinal injury score was also significantly reduced, all of which suggested that emodin can attenuate CLP-induced intestinal epithelial barrier damage in sepsis. The DAO activity and urine $\mathrm{L} / \mathrm{M}$ ratio are important indicators for the clinical detection of increased permeability of the intestinal wall (36). After emodin pre-treatment, DAO activity in the blood and the L/M ratio in the urine showed a downward trend compared with the CLP treatment group alone, and this trend was more obvious at $24 \mathrm{~h}$. This suggested that emodin may inhibit the increase in intestinal permeability in septic rats and maintain the integrity of the intestinal epithelial TJ barrier.

The intact intestinal epithelial TJ barrier plays a key role in sepsis (37). The barrier can prevent intestinal pathogens from entering the intestinal tract through the paracellular pathway. The integrity of the intestinal epithelial TJ barrier is maintained by an intercellular junction complex formed by TJs, adhesion junctions, desmosomes and gap junctions (38). These ligation complexes form a semi-permeable diffusion barrier between individual cells. They can prevent the abnormal passage of antigens, solutes and water. TJs provide the backbone of the structural integrity of the barrier and also provide a physical basis for the epithelial barrier to ions and solutes (39). TJs are located at the apical ends of the lateral membrane of epithelial cells (40) and have been shown to contain at least 40 proteins (9), such as claudins, ZO-1 and occludin (41). There are two main ways to regulate TJ transport, the pore pathway and the leak pathway (42). The first pathway is characterized by a small pore, which is thought to be created by claudins. The pore pathway is closely related to claudin protein activity (43) and allows the passage of small solutes and the transport of specific ions. Different claudin proteins have different functions. In particular, claudin-3, a barrier-forming protein, has no charge selectivity and is ubiquitously expressed in the jejunum, ileum and colon of rats (44). Previous studies suggested that the permeability of the pore pathway can directly regulate myosin light chain kinase (MLCK) by tumour necrosis factor (TNF)- $\alpha$ (45). When sepsis occurs, TNF- $\alpha$ regulates the upregulation of MLCK promoter activity through the $\mathrm{NF}-x \mathrm{~B}$ signalling pathway, thereby inducing increased expression of MLCK (46). Increased expression of MLCK can further promote increases in the permeability of the pore pathway, which affects the expression of claudin proteins (42). Garcia-Hernandez et al (47), found that claudin-3 was expressed at lower levels in inflammatory bowel disease. Haines et al (19), found that the protein expression levels of claudin-3 were positively correlated with intestinal epithelial tightness. In the present study, immunohistochemistry, western blotting and RT-qPCR were used to analyse the expression profile of claudin-3 in intestinal tissues. The results showed that the grading grey scales of claudin-3 protein and the mRNA levels of claudin-3 were lower in the CLP group compared with those of the corresponding sham group, with a significant difference at $24 \mathrm{~h}$. The low expression of claudin-3 in the CLP model group is consistent with that of existing research (48). These results indicated that CLP may reduce intestinal barrier integrity by reducing the expression of the barrier-forming protein claudin-3.
The leak pathway controls the paracellular transport of large uncharged solutes (proteins and bacterial LPS but not bacterial cells). Under normal physiological conditions, LPS in the intestinal lumen has no effect on intestinal wall integrity. When sepsis occurs, LPS and inflammatory cytokines (such as TNF- $\alpha$ ) in the intestinal lumen can enter the body through the leak pathway (47). ZO-1 and occludin are related to the leak pathway and are both involved in the maintenance and regulation of the leakage channel flux barrier. ZO-1 is critical for the assembly of TJs and epithelial barrier functions. Claudins and occludin proteins are linked to cytoskeletal actomyosin fibres by members of the ZO-1 protein family (49). In addition to providing a bridge between occludin and claudins and the cytoskeleton, ZO-1 may also affect the expression levels of transmembrane proteins at the transcriptional and post-translational levels (50). Occludin, a TJ protein, is mainly located in the TJs of cells after phosphorylation. When occludin is abnormally distributed and expressed in the structure of the small intestine, there is an increase in the intercellular space and an increase in permeability between endothelial cells (31). Zhang et al (51), found that the expression levels of the intestinal epithelial TJ proteins, ZO-1 and occludin, were both decreased in an LPS-induced sepsis model. In the present study, immunohistochemical staining, western blotting and RT-qPCR were used to detect the expression profile of ZO-1 and occludin in the intestinal epithelial barrier in sepsis. The grey scales of ZO-1 protein showed a downward trend in the CLP group, and there was a significant difference at $12 \mathrm{~h}$. The mRNA levels of ZO-1 significantly decreased in the CLP group at $24 \mathrm{~h}$, but were increased at $12 \mathrm{~h}$. This phenomenon also occurs in different tissues of other animal models (52). The decrease in $\mathrm{ZO}-1$ protein at $12 \mathrm{~h}$ could not be explained by a reduction in ZO-1 transcription, which may be a secondary factor to some combination of posttranslational regulation or degradation (52). However, occludin protein expression did not change during sepsis, but mRNA levels showed an upward trend. It is possible that occludin protein is modulated via post-transcriptional or post-translational regulations (53).

Decreased expression levels of TJ proteins are strongly associated with impaired intestinal barrier function (54). Thus, the protective effect of emodin against CLP-induced intestinal barrier injury can be partially explained by the increased expression of $\mathrm{TJ}$ proteins. In recent years, numerous scientific reports described that emodin has an anti-inflammatory effect and can reduce the expression of the inflammatory factor TNF- $\alpha$ (55-57). Compared with expression in the corresponding CLP group, the protein expression levels of claudin-3 in the CLP + emodin group showed a continuous and uniform distribution, with a smoother edge and an enhanced positive signal. In addition, the protein and mRNA expression levels of claudin-3 were significantly increased in the CLP + emodin group compared with the CLP alone treatment group. It is possible that emodin can reduce TNF- $\alpha$ levels in the sepsis model (58) and further inhibit MLCK activation. Further studies are required to clarify the detailed mechanisms of action. These data indicated that emodin may inhibit the increase in intestinal barrier permeability by promoting the transcription and translation of the TJ protein claudin-3. 
The present study also found that in the emodin pre-treatment group, expression of ZO-1 and occludin proteins showed a continuous and uniform distribution, with a smoother edge and an enhanced positive signal, when compared with that of the corresponding CLP group. Moreover, protein and mRNA expression levels of ZO-1 were significantly increased in the CLP + emodin group. However, while the expression of occludin protein was increased, the mRNA was not. Although studies have found that occludin overexpression is protective in Madin-Darby canine kidney cells in vitro (59), in vivo studies indicate that occludin gene defects do not affect the integrity of intestinal epithelial TJs (60). Therefore, further investigation is needed to determine whether occludin plays a role in intestinal epithelial paracellular permeability. Together, these findings suggested that emodin may inhibit the increase in intestinal barrier permeability by promoting the transcription and translation of $\mathrm{ZO}-1$ and occludin proteins, at least to a certain degree.

In conclusion, emodin may alleviate intestinal barrier damage caused by CLP, inhibit the increase in intestinal barrier permeability and maintain the integrity of the intestinal epithelial barrier. Intestinal epithelial TJ proteins are key molecules for the determination of intestinal epithelial barrier permeability. Based on results from previous studies and the present study, it is speculated that the amelioration of intestinal epithelial barrier damage by emodin can be attributed, at least in part, to the promotion of claudin-3, ZO-1 and occludin expression. However, the specific mechanism of action requires further exploration. In the present study, other TJ proteins were not tested, and the effect of emodin on other TJ proteins is not fully understood. Despite these limitations, the protective effect of emodin on CLP-induced intestinal epithelial TJ barrier injury was investigated and an understanding of the protective effect of emodin on regulating intestinal barrier dysfunction in sepsis was provided.

\section{Acknowledgements}

Not applicable.

\section{Funding}

The present study was supported by funding from the National Natural Science Foundation of China (grant no. 81573901$)$.

\section{Availability of data and material}

The datasets used and analyzed during the present study are available from the corresponding author on reasonable request.

\section{Authors' contributions}

YL, RG and YS designed experiments and analyzed and interpreted data. YL, RG, MZ, PC and JL performed experiments and collected data. YS and $\mathrm{RG}$ reviewed articles and contributed reagents and materials. YL wrote the manuscript. All authors read and approved the final manuscript.

\section{Ethics and approval}

This study was approved by the Ethics Committee of Putuo Hospital affiliated with Shanghai University of Traditional Chinese Medicine. All animal experiments were in accordance with the Experimental Animal Centre Committee of Putuo Hospital (certification no. SYNK 2018-0032).

\section{Patient consent for publication}

Not applicable.

\section{Competing interests}

The authors declare that they have no competing interests.

\section{References}

1. Singer M, Deutschman CS, Seymour CW, Shankar-Hari M, Annane D, Bauer M, Bellomo R, Bernard GR, Chiche JD, Coopersmith CM, et al: The third international consensus definitions for sepsis and septic shock (Sepsis-3). JAMA 315: 801-810, 2016.

2. Condotta SA, Cabrera-Perez J, Badovinac VP and Griffith TS: T-cell-mediated immunity and the role of TRAIL in sepsis-induced immunosuppression. Crit Rev Immunol 33: 23-40, 2013.

3. Cao Y, Chen Q, Wang Z, Yu T, Wu J, Jiang X, Jin X and Lu W: PLK1 protects against sepsis-induced intestinal barrier dysfunction. Sci Rep 8: 1055, 2018.

4. Hua S, Liu X, Lv S and Wang Z: Protective effects of cucurbitacin $\mathrm{B}$ on acute lung injury induced by sepsis in rats. Med Sci Monit 23: 1355-1362, 2017.

5. Liu W, Wang XH, Yang XJ, Zhang XY and Qi WJ: Intestinal barrier dysfunction and its related factors in patients with sepsis. Zhonghua Yi Xue Za Zhi 96: 3568-3572, 2016 (In Chinese).

6. Yoseph BP, Klingensmith NJ, Liang Z, Breed ER, Burd EM, Mittal R, Dominguez JA, Petrie B, Ford ML and Coopersmith CM: Mechanisms of intestinal barrier dysfunction in sepsis. Shock 46: 52-59, 2016.

7. Xu J, Liu Z, Zhan W, Jiang R, Yang C, Zhan H and Xiong Y: Recombinant TsP53 modulates intestinal epithelial barrier integrity via upregulation of ZO-1 in LPS-induced septic mice. Mol Med Rep 17: 1212-1218, 2018

8. Lu WH, Jin XJ, Jiang XG, Wang Z, Wu JY and Shen GG: Resuscitation with hydroxyethyl starch 130/0.4 attenuates intestinal injury in a rabbit model of sepsis. Indian J Pharmacol 47: 49-54, 2015.

9. Li Q, Zhang Q, Wang C, Liu X, Li N and Li J: Disruption of tight junctions during polymicrobial sepsis in vivo. J Pathol 218: 210-221, 2009.

10. Gu L, Li N, Gong J, Li Q, Zhu W and Li J: Berberine ameliorates intestinal epithelial tight-junction damage and down-regulates myosin light chain kinase pathways in a mouse model of endotoxinemia. J Infect Dis 203: 1602-1612, 2011.

11. Wang L, Cui YL, Zhang Z, Lin ZF and Chen DC: Rhubarb monomers protect intestinal mucosal barrier in sepsis via junction proteins. Chin Med J (Engl) 130: 1218-1225, 2017.

12. Li MX, Liu JF, Lu JD, Zhu Y, Kuang DW, Xiang JB, Sun P, Wang W, Xue J, Gu Y, et al: Plasmadiafiltration ameliorating gut mucosal barrier dysfunction and improving survival in porcine sepsis models. Intensive Care Med Exp 4: 31, 2016.

13. Chen G, Huang B, Fu S, Li B, Ran X, He D, Jiang L, Li Y, Liu B, Xie L, et al: $\mathrm{G}$ protein-coupled receptor $109 \mathrm{~A}$ and host microbiota, odulate intestinal epithelial integrity during sepsis. Front Immunol 9: 2079, 2018.

14. Chen DC and Wang L: Mechanisms of therapeutic effects of rhubarb on gut origin sepsis. Chin J Traumatol 12: 365-369, 2009.

15. Li P, Lu Q, Jiang W, Pei X, Sun Y, Hao H and Hao K: Pharmacokinetics and pharmacodynamics of rhubarb anthraquinones extract in normal and disease rats. Biomed Pharmacother 91: 425-435, 2017. 
16. Wang G, Sun B, Zhu H, Gao Y, Li X, Xue D and Jiang H: Protective effects of emodin combined with danshensu on experimental severe acute pancreatitis. Inflamm Res 59: 479-488, 2010.

17. Chen G, Zhang J, Zhang H, Xiao Y, Kao X, Liu Y and Liu Z: Anti-inflammatory effect of emodin on lipopolysaccharide-induced keratitis in Wistar rats. Int J Clin Exp Med 8: 12382-12389, 2015.

18. Ning JW, Zhang Y, Yu MS, Gu ML, Xu J, Usman A and Ji F: Emodin alleviates intestinal mucosal injury in rats with severe acute pancreatitis via the caspase-1 inhibition. Hepatobiliary Pancreat Dis Int, 2017. 16(4): p. 431-436.

19. Haines RJ, Beard RS Jr, Chen L, Eitnier RA and Wu MH: Interleukin-1beta mediates beta-catenin-driven downregulation of claudin-3 and barrier dysfunction in Caco2 cells. Dig Dis Sci 61: 2252-2261, 2016.

20. Holmes JL, Van Itallie CM, Rasmussen JE and Anderson JM Claudin profiling in the mouse during postnatal intestinal development and along the gastrointestinal tract reveals complex expression patterns. Gene Expr Patterns 6: 581-588, 2006.

21. Rahner C, Mitic LL and Anderson JM: Heterogeneity in expression and subcellular localization of claudins $2,3,4$, and 5 in the rat liver, pancreas, and gut. Gastroenterology 120: 411-422, 2001.

22. Sun Y, Sun L, Liu S, Song J, Cheng J and Liu J: Effect of emodin on Aquaporin 5 expression in rats with sepsis-induced acute lung injury. J Tradit Chin Med 35: 679-684, 2015.

23. Pereira RS, Bertoncheli CM, Adefegha SA, Castilhos LG, Silveira KL, Rezer JFP, Doleski PH, Abdalla FH, Santos KF, Leal CAM, et al: Sepsis induced by cecal ligation and perforation (CLP) alters nucleotidase activities in platelets of rats. Microb Pathog 111: 345-351, 2017.

24. Shi X, Zhang Y, Wang $\mathrm{H}$ and Zeng S: Effect of triggering receptor expressed on myeloid cells 1 (TREM-1) blockade in rats with cecal ligation and puncture (CLP)-induced sepsis. Med Sci Monit 23: 5049-5055, 2017

25. Wu J, Lyu B, Gan T, Wang L and Zhu M: Electroacupuncture improves acute bowel injury recovery in rat models. Exp Ther Med 14: 4655-4662, 2017

26. Guo S, Nighot M, Al-Sadi R, Alhmoud T, Nighot P and Ma TY: Lipopolysaccharide regulation of intestinal tight junction permeability is mediated by TLR4 signal transduction pathway activation of FAK and MyD88. J Immunol 195: 4999-5010, 2015.

27. Livak KJ and Schmittgen TD: Analysis of relative gene expression data using real-time quantitative PCR and the 2(-Delta Delta C(T)) Method. Methods 25: 402-408, 2001.

28. Zhang B, Liu C, Yang N and Wang X: A comparative study of changes of autophagy in rat models of CLP versus LPS induced sepsis. Exp Ther Med 14: 2194-2200, 2017.

29. Gao M, Jiang Y, Xiao X, Peng Y, Xiao X and Yang M: Protective effect of pioglitazone on sepsis-induced intestinal injury in a rodent model. J Surg Res 195: 550-558, 2015

30. Zhou H, Liang H, Li ZF, Xiang H, Liu W and Li JG: Vagus nerve stimulation attenuates intestinal epithelial tight junctions disruption in endotoxemic mice through $\alpha 7$ nicotinic acetylcholine receptors. Shock 40: 144-151, 2013.

31. Yang J, Zhang S, Wu J, Zhang J, Dong J, Guo P, Tang S, Zhang W and $\mathrm{Wu} F$ : Imipenem and normal saline with cyclophosphamide have positive effects on the intestinal barrier in rats with sepsis Biomed Pap Med Fac Univ Palacky Olomouc Czech Repub 162: 90-98, 2018.

32. Fang H, Gong C, Fu J, Liu X, Bi H, Cheng Y, Liu Y, Tang Y and Wang D: Evaluation of 2 rat models for sepsis developed by improved cecal ligation/puncture or feces intraperitoneal-injection. Med Sci Monit 26: e919054, 2020.

33. Chen J, Huang C, Wang J, Zhou H, Lu Y, Lou L, Zheng J, Tian L, Wang X, Cao Z, et al: Dysbiosis of intestinal microbiota and decrease in paneth cell antimicrobial peptide level during acute necrotizing pancreatitis in rats. PLoS One 12: e0176583, 2017.

34. Warners MJ, Vlieg-Boerstra BJ, Verheij J, van Hamersveld PHP, van Rhijn BD, Van Ampting MTJ, Harthoorn LF, de Jonge WJ, Smout AJPM and Bredenoord AJ: Esophageal and small intestinal mucosal integrity in eosinophilic esophagitis and response to an elemental diet. Am J Gastroenterol 112: 1061-1071, 2017.

35. Chen YK, Xu YK, Zhang H, Yin JT, Fan X, Liu DD, Fu HY and Wan B: Emodin alleviates jejunum injury in rats with sepsis by inhibiting inflammation response. Biomed Pharmacother 84 : 1001-1007, 2016.

36. Xu GF, Guo M, Tian ZQ, Wu GZ, Zou XP and Zhang WJ: Increased of serum high-mobility group box chromosomal protein 1 correlated with intestinal mucosal barrier injury in patients with severe acute pancreatitis. World J Emerg Surg 9: $61,2014$.
37. Cuellar P, Hernández-Nava E, García-Rivera G, Chávez-Munguía B, Schnoor M, Betanzos A and Orozco E: Entamoeba histolytica EhCP112 dislocates and degrades claudin-1 and claudin-2 at tight junctions of the intestinal epithelium. Front Cell Infect Microbiol 7: 372, 2017.

38. Chen J, Zhang R, Wang J, Yu P, Liu Q, Zeng D, Song H and Kuang Z: Protective effects of baicalin on LPS-induced injury in intestinal epithelial cells and intercellular tight junctions. Can J Physiol Pharmacol 93: 233-237, 2015.

39. ColganSP,Curtis VF,LanisJM and GloverLE: Metabolicregulation of intestinal epithelial barrierduring inflammation. Tissue Barriers 3: e970936,2015.

40. Xing T, Camacho Salazar R and Chen YH: Animal models for studying epithelial barriers in neonatal necrotizing enterocolitis, inflammatory bowel disease and colorectal cancer. Tissue Barriers 5: e1356901, 2017.

41. Salvo Romero E, Alonso CotonerC,Pardo Camacho C,Casado Bedmar $\mathrm{M}$ and Vicario M: The intestinal barrier function and its involvement in digestive disease. Rev Esp Enferm Dig 107: 686-696, 2015.

42. Turner JR: Intestinal mucosal barrier function in health and disease. Nat Rev Immunol 9: 799-809, 2009.

43. Shen L, Weber CR, Raleigh DR, Yu D and Turner JR: Tight junction pore and leak pathways: A dynamic duo. Annu Rev Physiol 73: 283-309, 2011.

44. Barmeyer C, Fromm M and Schulzke JD: Active and passive involvement of claudins in the pathophysiology of intestinal inflammatory diseases. Pflugers Arch 469: 15-26, 2017.

45. Dokladny K, MN Zuhl and PL Moseley: Intestinal epithelial barrier function and tight junction proteins with heat and exercise. J Appl Physiol (1985) 120 (6): 692-701, 2016.

46. Zhang $\mathrm{Y}$ and $\mathrm{Li} \mathrm{J}$ : Carbachol ameliorates lipopolysaccharide-induced intestinal epithelial tight junction damage by down-regulating NF- $x \beta$ and myosin light-chain kinase pathways. Biochem Biophys Res Commun 428: 321-326, 2012.

47. Garcia-Hernandez V, Quiros $M$ and Nusrat A: Intestinal epithelial claudins: Expression and regulation in homeostasis and inflammation. Ann N Y Acad Sci 1397: 66-79, 2017.

48. Xing X, Jiang R, Wang L, Lei S, Zhi Y, Wu Y, Zhu M, Huang L, $\mathrm{Xia} G$ and Chen Z: Shenfu injection alleviates intestine epithelial damage in septic rats. Am J Emerg Med 33: 1665-1670, 2015.

49. Sonika U, Goswami P, Thakur B, Yadav R, Das P, Ahuja V and Saraya A: Mechanism of increased intestinal permeability in acute pancreatitis: Alteration in tight junction proteins. J Clin Gastroenterol 51: 461-466, 2017.

50. Li W, Sun K, Ji Y, Wu Z, Wang W, Dai Z and Wu G: Glycine regulates expression and distribution of claudin-7 and ZO-3 proteins in intestinal porcine epithelial cells. J Nutr 146: 964-969, 2016.

51. Zhang S, Zheng S, Wang X, Shi Q, Wang X, Yuan S, Wang G and Ji Z: Carbon monoxide-releasing molecule-2 reduces intestinal epithelial tight-junction damage and mortality in septic rats. PLoS One 10: e0145988, 2015.

52. Eadon MT, Hack BK, Xu C, Ko B, Toback FG and Cunningham PN: Endotoxemia alters tight junction gene and protein expression in the kidney. Am J Physiol Renal Physiol 303: F821-F830, 2012.

53. Hwang I, An BS, Yang H, Kang HS, Jung EM and Jeung EB Tissue-specific expression of occludin, zona occludens-1, and junction adhesion molecule $\mathrm{A}$ in the duodenum, ileum, colon, kidney, liver, lung, brain, and skeletal muscle of C57BL mice. J Physiol Pharmacol 64: 11-18, 2013.

54. Yan $\mathrm{H}$ and Ajuwon KM: Butyrate modifies intestinal barrier function in IPEC-J2 cells through a selective upregulation of tight junction proteins and activation of the Akt signaling pathway. PLoS One 12: e0179586,2017.

55. Chen D, Liu J, Lu L, Huang Y, Wang Y, Wang M, Liu Y, Xie D, Chen J, Diao J, et al: Emodin attenuates TNF- $\alpha$-induced apoptosis and autophagy in mouse $\mathrm{C} 2 \mathrm{C} 12$ myoblasts though the phosphorylation of Akt. Int Immunopharmacol 34: 107-113, 2016.

56. Xia S, Ni Y, Zhou Q, Liu H, Xiang H, Sui H and Shang D: Emodin attenuates severe acute pancreatitis via antioxidant and anti-inflammatory activity. Inflammation 42: 2129-2138, 2019.

57. Dong Y, Zhang L, Jiang Y, Dai J, Tang L and Liu G: Emodin reactivated autophagy and alleviated inflammatory lung injury in mice with lethal endotoxemia. Exp Anim 68: 559-568, 2019.

58. Xia XM, Li BK, Xing SM and Ruan HL: Emodin promoted pancreatic claudin-5 and occludin expression in experimental acute pancreatitis rats. World J Gastroenterol 18: 2132-2139, 2012.

59. Cummins PM: Occludin: One protein, many forms. Mol Cell Biol 32: 242-250, 2012.

60. Van Itallie CM and Anderson JM: Claudin interactions in and out of the tight junction. Tissue Barriers 1: e25247, 2013.

This work is licensed under a Creative Commons

Attribution-NonCommercial-NoDerivatives 4.0 International (CC BY-NC-ND 4.0) License. 